\title{
Managing hyperparathyroidism in hemodialysis: role of etelcalcetide
}

\author{
Keith E Eidman' \\ James B Wetmore ${ }^{1,2}$ \\ 'Division of Nephrology, Hennepin \\ County Medical Center, University of \\ Minnesota, Minneapolis, MN, ${ }^{2} \mathrm{Chronic}$ \\ Disease Research Group, Minneapolis \\ Medical Research Foundation, \\ Minneapolis, MN, USA
}

This article was published in the following Dove Press journal: International Journal of Nephrology and Renovascular Disease

Correspondence: James B Wetmore Chronic Disease Research Group, Minneapolis Medical Research Foundation, 70I Park Avenue, Suite S4.100, Minneapolis, MN 55415, USA $\mathrm{Tel}+\mathrm{I} 6128736988$

Fax + 6128731644

Email James.Wetmore@hcmed.org

\begin{abstract}
Secondary hyperparathyroidism (SHPT) is common in patients receiving maintenance hemodialysis and is associated with adverse outcomes. Currently, SHPT is managed by reducing circulating levels of phosphate with oral binders and parathyroid hormone (PTH) with vitamin D analogs and/or the calcimimetic cinacalcet. Etelcalcetide, a novel calcimimetic administered intravenously (IV) at the end of a hemodialysis treatment session, effectively reduces PTH in clinical trials when given thrice weekly. Additional clinical effects include reductions in circulating levels of phosphate and FGF-23 and an improved profile of markers of bone turnover. However, despite being administered IV, etelcalcetide appears to be associated with rates of nausea and vomiting comparable to those of cinacalcet. Additionally, etelcalcetide, relative to placebo, causes hypocalcemia and prolonged electrocardiographic QT intervals, effects that must be considered when contemplating its use. Etelcalcetide likely has a role in treating hemodialysis patients with uncontrolled SHPT or with hypercalcemia or hyperphosphatemia receiving activated vitamin D compounds. However, its use should be at least partially constrained by consideration of the risk of hypocalcemia and resultant prolonged QT intervals in vulnerable patients. Because of its effectiveness as a PTH-reducing agent administered in the dialysis unit, etelcalcetide represents a potentially promising new therapeutic approach to the often vexing problem of SHPT in hemodialysis patients. However, whether its use is associated with changes in surrogate clinical end points, such as effects on rates of parathyroidectomy, fracture, vascular calcification, or mortality or on quality of life, remains to be studied.
\end{abstract}

Keywords: chronic kidney disease, calcimimetic, parathyroidectomy

\section{Introduction}

Abnormalities in calcium, phosphorous, and parathyroid hormone (PTH), hallmarks of the condition known as chronic kidney disease - mineral and bone disorder (CKDMBD), ${ }^{1}$ are associated with adverse outcomes in patients receiving maintenance dialysis. $^{2-4}$ In patients with advanced chronic kidney disease (CKD), development of secondary hyperparathyroidism (SHPT) contributes to CKD-MBD 5 and results in derangements of circulating markers of mineral metabolism and in dysregulation of skeletal and cardiovascular physiology. ${ }^{6-8}$ Epidemiologic studies of dialysis patients provide substantial evidence that elevated PTH is associated with mortality. ${ }^{3,4}$ However, therapies targeting abnormal CKD-MBD parameters, while improving biochemical end points, ${ }^{9}$ have failed to convincingly demonstrate reductions in "hard" end points such as all-cause and cardiovascular mortality in clinical trials. ${ }^{10}$ Thus, SHPT management currently centers chiefly on therapies targeting serum phosphate reduction through 
dietary phosphate restriction and oral phosphate binders and control of PTH via vitamin D analogs and calcimimetics. ${ }^{11}$

Vitamin D analogs such as paricalcitol and doxercalciferol, longtime cornerstones of SHPT therapy, effectively reduce PTH in most patients with SHPT but often result in elevated calcium and phosphorous levels, ${ }^{12}$ a serious impediment to their use. Additionally, vitamin D analogs elevate circulating levels of FGF-23, ${ }^{13}$ a marker associated with increased mortality in epidemiologic studies. ${ }^{14}$ Dissatisfaction with traditional treatment options prompted exploration of a novel mechanism for SHPT treatment that targeted the calcium-sensing receptor (CaSR). These efforts culminated in the introduction of cinacalcet, an orally administered allosteric modulator of the CaSR, in 2004. Cinacalcet was met with initial enthusiasm by the nephrology community.$^{15}$ However, for a variety of reasons that remain only partially understood, it was not deployed as long-term maintenance therapy to the hoped for or expected degree.

To improve the management of SHPT, development of an intravenously (IV) administered calcimimetic was undertaken. An IV calcimimetic was hypothesized to have the potential to overcome two major barriers to cinacalcet use, namely, nonadherence and gastrointestinal side effects. Developed over the past decade, etelcalcetide is an IV agent administered via the dialysis circuit during the hemodialysis treatment session. Later, we discuss the development of calcimimetics in general and etelcalcetide in particular, evidence for etelcalcetide's utility and safety concerns related to it, and potential clinical scenarios for etelcalcetide use in the treatment of SHPT in patients receiving maintenance hemodialysis.

\section{History of calcimimetics}

Cinacalcet, a first-in-class allosteric modulator of the CaSR, proved very effective at reducing PTH levels in clinical trials. ${ }^{9}$ In addition, its use, relative to use of vitamin $\mathrm{D}$ analogs, was associated with reduced circulating phosphate levels and, in later analyses, of FGF $23 .{ }^{13}$ Approved for use in the US in 2004, cinacalcet was quickly and widely adopted in practice. One US cohort study reported $10 \%$ prevalence of use within 6 months of drug approval, rising to $32 \%$ within 2 years. ${ }^{15}$ Not surprisingly, use patterns have subsequently varied over time, related to varying worldwide practice patterns and to differences in guidelines and recommendations for PTH control. ${ }^{16}$

Whether cinacalcet might be associated with a mortality benefit was tested in the most ambitious clinical trial ever undertaken in nephrology. The Evaluation of Cinacalcet Hydrochloride Therapy to Lower Cardiovascular Events (EVOLVE) trial enrolled $\sim 3900$ patients to ascertain whether, compared with placebo, cinacalcet would reduce deaths plus nonfatal cardiovascular events in patients receiving maintenance dialysis. In this event-driven trial of $>5$ years duration, cinacalcet was not associated with a reduction in the primary end point $(P=0.11) .{ }^{10}$ However, a range of additional prespecified analyses, including multivariable-adjusted, astreated, lag-censored, and inverse probability-of-censoringweighted analyses, suggested benefit. This discordance in findings led to robust debate in the nephrology community as to whether cinacalcet can truly confer morbidity and mortality benefits to patients when used in real-world scenarios. ${ }^{17,18}$

Even in the absence of a mortality benefit, cinacalcet was hypothesized to have a role as a "parathyroidectomy (PTX)sparing therapy". Initially, "ecological" evidence suggested that introduction of cinacalcet was associated with lower PTX rates, ${ }^{19}$ a finding that presaged a key secondary end point from EVOLVE, namely, that use of cinacalcet significantly reduced the likelihood of PTX. However, because cinacalcet is associated with hypocalcemia (an effect widely anticipated based on early clinical trial results) and with gastrointestinal side effects (chiefly nausea and vomiting), consistent exposure has proved challenging in clinical practice, limiting its effectiveness. This is the case not only in real-world settings but also in the EVOLVE trial itself, in which $62.0 \%$ of patients randomized to cinacalcet discontinued the drug during the study. This lack of consistent exposure among many cinacalcet-treated patients, along with liberalization of the PTH goal range suggested by Kidney Disease: Improving Global Outcomes (KDIGO) in $2009,{ }^{1}$ may explain why PTX rates have not declined in recent years in many countries, including the US. ${ }^{20}$ A notable exception is Japan, which has experienced a sustained decline in PTX rates. ${ }^{21}$ Given that PTX is often associated with suboptimal short-term outcomes, ${ }^{22,23}$ whether new-generation calcimimetics can be used as PTX-sparing therapy is an important question.

\section{Molecular actions and pharmacokinetics of etelcalcetide}

While both cinacalcet and etelcalcetide affect the CaSR, they differ in structure, molecular interactions on the CaSR, mode of delivery, half-life, and potential for drug interactions via the cytochrome P450 system. Etelcalcetide, an agonist of the human CaSR, is a novel synthetic peptide consisting of only eight amino acids. It comprises a main chain of seven linear D-amino acids with a side-chain of cysteine residue, 
linked via a disulfide bond, in the L-configuration. It has a molecular weight of $\sim 1048 \mathrm{Da}$ and is positively charged at $\mathrm{pH} 7.4 .^{24} \mathrm{~A} \mathrm{D}$-amino acid property is that they are more resistant to proteolytic degradation and less likely to generate an immune response (i.e., antibodies) than L-amino acids. ${ }^{25} \mathrm{As}$ there are relatively few D-amino acid-based pharmaceutical compounds, attaining full understanding of the pharmacodynamics and pharmacokinetics of this molecule during development and testing was especially important.

The interaction site of etelcalcetide with the human CaSR was elucidated by studying the differential effects of the peptide in various mammalian systems. Like all G-protein-coupled receptors, the CaSR contains extracellular, transmembrane, and intracellular domains. The extracellular domain comprises two subdomains, a Venus flytrap domain and a carboxy-terminal cysteine-rich domain. ${ }^{26}$ The sequence of the former domain differs by species; pigs have a tyrosine residue at position 482, while human beings and several other mammalian species have cysteine. ${ }^{27}$ Lack of pharmacodynamic activity in pigs permitted Alexander et $\mathrm{al}^{27}$ to demonstrate that covalent disulfide bond formation between the cysteine at position 482 on the human CaSR and the cysteine residue from etelcalcetide are critical to pharmacological activity of the peptide. Stimulation of the CaSR by etelcalcetide requires cysteines in both the agonist peptide and the CaSR, and the extent of the interaction correlates with activity.

As a novel predominately D-amino acid molecule, etelcalcetide required extensive study regarding its metabolism and disposition in vivo. After a single IV administration, etelcalcetide appears to be rapidly distributed. It is biotransformed via disulfide exchange of the L-cysteine with circulating thiols, including serum albumin. ${ }^{28}$ Notably, there appears to be little to no risk for P450- or transporter-mediated drug interactions. In human beings with intact renal function, disposition is predominately via renal elimination. ${ }^{24}$ In human beings without renal function, disposition and elimination were tested with C-14-radiolabeled etelcalcetide. After a single dose, thrice-weekly hemodialysis was estimated to account for $\sim 60 \%$ of elimination, nonspecific elimination to account for $30 \%$, gastrointestinal tract to account for $6 \%$, and urine to account for $<3 \% .{ }^{29}$ Relatively rapid removal by hemodialysis therefore necessitates administration at the end of the hemodialysis treatment session. ${ }^{30}$

Nonclinical safety data via multiple techniques failed to demonstrate any significant safety signals, with no apparent evidence of mutagenicity or genotoxicity. While preexisting and developing etelcalcetide antibodies can be detected, no apparent clinical consequences have been shown. ${ }^{31}$ The only adverse effects seen in animal studies involved effects and sequelae of hypocalcemia.

\section{Preclinical studies of etelcalcetide}

In nonhuman in vivo studies, etelcalcetide was shown to inhibit PTH secretion in animals with intact kidney function and with CKD, such as the rat 5/6th nephrectomy model. ${ }^{32}$ Further animal studies in uremic rats revealed affects beyond reduced PTH levels, including attenuated parathyroid gland hyperplasia, reduced vascular calcification, and increased expression of receptors for FGF23 and vitamin D, ${ }^{33}$ suggesting that etelcalcetide could treat SHPT-related laboratory abnormalities and might conceivably alter the natural history of CKD-MBD.

\section{Phase I and II studies of etelcalcetide}

The original Phase I study of etelcalcetide involved 32 healthy participants randomized to a single dose of $0.5,2$, 5 , or $10 \mathrm{mg} .{ }^{34}$ The pharmacokinetics of etelcalcetide demonstrated what is known as an open three-compartment model with first-order elimination. The large volume of distribution (>790 L, according to the US Food and Drug Administration [FDA]-approved package insert) suggests potential peripheral distribution and apparent reversible binding. Etelcalcetide's half-life in these healthy participants was $\sim 20.5$ hours.

Following single-dose administration, effects of etelcalcetide demonstrated an acute PTH reduction within $10 \mathrm{~min}$ utes, a nadir by 1 hour, and a return toward baseline by 24 hours. ${ }^{35}$ Decreases in calcium were dose dependent and lagged behind changes in PTH by several hours, reaching a nadir 10-15 hours after infusion. An additional effect in this healthy cohort included a substantial $(>30 \%)$ reduction in FGF23 levels at the highest dose tested $(10 \mathrm{mg}){ }^{35}$

An early single-dose clinical study involving participants receiving hemodialysis broadly confirmed observations in animal studies, namely, a dose-dependent reduction in PTH and calcium and, to a lesser degree, FGF23. In contrast to cinacalcet, etelcalcetide demonstrated prolonged suppression of PTH. ${ }^{36}$ Similar results were demonstrated in a Phase I hemodialysis cohort from Japan, in which PTH suppression was also shown to be sustained during the interdialytic period when using multiple doses. ${ }^{37}$

Phase II studies further demonstrated the safety and efficacy of etelcalcetide. ${ }^{38,39}$ For example, Bushinsky et al conducted a 12-week dose-titration efficacy study followed by an open-label extension for safety in 37 participants on 
hemodialysis. Etelcalcetide was administered IV thrice weekly starting at a $5 \mathrm{mg}$ dose and titrated based on PTH and albumin-corrected serum calcium levels to target a relatively narrow PTH range of 150-300 pg/mL. The mean decrease in PTH was $53.6 \%$, with $89.0 \%$ of participants achieving a $30 \%$ or greater reduction from baseline. Overall, etelcalcetide was characterized as being well tolerated. ${ }^{39}$ Broadly, similar findings were reported by Bell et al, ${ }^{38}$ who tested 5 or $10 \mathrm{mg}$ doses against placebo for 2-4 weeks; $90 \%$ of participants with PTH $<600 \mathrm{pg} / \mathrm{mL}$ achieved a $30 \%$ or greater reduction in PTH, as did $64 \%$ of participants with PTH $\geq 600 \mathrm{pg} / \mathrm{mL}$.

Conceptually, similar Phase II trials were conducted in Japanese hemodialysis patients. ${ }^{37,40}$ In a 12 -week dosetitration trial, etelcalcetide was initially dosed at $5 \mathrm{mg}$ and then titrated every 4 weeks to 10 or $15 \mathrm{mg}$. Importantly, with different guidelines for treating CKD-MBD and a PTH goal different from that endorsed by KDIGO, the parameters of this Japanese study differed relative to studies conducted in the US; the baseline PTH at initiation was only $538 \pm 188 \mathrm{pg} /$ $\mathrm{mL}$, and doses were increased when PTH exceeded $240 \mathrm{pg} /$ $\mathrm{mL}$. However, a robust decrease in PTH of $65.9 \%$ was again demonstrated compared with baseline. Additional findings by the end of the treatment period included a dose-dependent decrease in calcium of $13.8 \%$, a $23.5 \%$ mean decrease in phosphorus, and a $14.4 \%$ mean decrease in FGF23 levels. Adverse events were common but were typically mild or moderate; $16.7 \%$ of patients experienced decreased calcium and $12.5 \%$ of patients experienced vomiting.

\section{Phase III trials of etelcalcetide}

Results of two nearly identical Phase III, multinational, randomized clinical trials, referred to as trials $\mathrm{A}$ and $\mathrm{B}$, were recently published in a single report. ${ }^{41}$ Both trials tested the effects of etelcalcetide versus placebo in patients with SHPT undergoing maintenance hemodialysis. Consistent with other trials, ${ }^{42}$ the primary end point was a $30 \%$ or greater reduction in PTH levels. The trials enrolled 1013 participants with PTH $>400 \mathrm{pg} / \mathrm{mL}$, stable doses of SHPT-related medications, and an albumin-corrected serum calcium level of $\geq 8.3 \mathrm{mg} /$ $\mathrm{dL}$; cinacalcet use was prohibited for 4 weeks preceding and during the study period. Patients were randomized to receive standard-of-care therapy alone (phosphate binders and activated vitamin D therapy, namely, calcitriol or its analogs) or standard therapies plus etelcalcetide. Patients received thrice-weekly dosing of etelcalcetide, administered as each hemodialysis session concluded. The starting dose was $5 \mathrm{mg}$ with dose titrations, based on PTH and albumincorrected calcium values, in 2.5 or $5 \mathrm{mg}$ increments at 4-week intervals; the maximum permitted dose was $15 \mathrm{mg}$. The trial consisted of a 20 -week dose-titration phase followed by a 7-week efficacy assessment phase, during which PTH values were averaged and compared between arms. In addition to the aforementioned primary end point, secondary end points included the proportion of patients with a mean PTH value of $\geq 300 \mathrm{pg} / \mathrm{mL}$ and percentage reductions in levels of PTH, calcium, phosphorus, and calcium-phosphorus product. Additional exploratory end points included changes in FGF23 levels and in markers of bone turnover, namely, bone-specific alkaline phosphatase and collagen type 1 crosslinked C-telopeptide. Participants were stratified by the PTH level, cinacalcet use within 8 weeks of randomization, and geographic region.

The recruited participants appeared broadly similar to the general US hemodialysis population; mean age was 58.2 years and $60.4 \%$ were men, $66.5 \%$ were white, and $27.9 \%$ were black. Baseline PTH levels were well randomized, with mean levels in both arms in trials $\mathrm{A}$ and $\mathrm{B}$ ranging from 820 to $852 \mathrm{pg} / \mathrm{mL}$. In the intention-to-treat analysis, results of trial A demonstrated a mean PTH decrease in etelcalcetide-treated participants from 849 to $384 \mathrm{pg} / \mathrm{mL}$ compared with an increase from 820 to $870 \mathrm{pg} / \mathrm{mL}$ in the placebo group. Of participants randomized to etelcalcetide, $74.0 \%$ achieved $\geq 30 \%$ reduction in $\mathrm{PTH}$ from baseline compared with only $8.3 \%$ in the placebo group $(P<0.001$ for the primary end point). Corresponding changes in $\mathrm{PTH}$ in trial B were $845-363 \mathrm{pg} / \mathrm{mL}$ in the treatment arm versus $852-960 \mathrm{pg} / \mathrm{mL}$ in the placebo arm; $75.3 \%$ participants in the treatment arm achieved $\geq 30 \%$ decrease in PTH compared to only $9.6 \%$ participants in the placebo arm $(P<0.001)$. Achievement of the primary end point was observed across all patient subgroups. Regarding absolute reduction of PTH, $49.6 \%$ of treated participants, versus $5.1 \%$ of placebo participants, achieved a PTH level of $<300 \mathrm{pg} / \mathrm{mL}(P<0.001)$ in trial A; corresponding values were $53.3 \%$ and $4.6 \%(P<0.001)$ in trial B. Notably, median per-session etelcalcetide dose differed by trial; $7.1 \mathrm{mg}$ for trial A and $5.0 \mathrm{mg}$ for trial B.

While secondary end points regarding percentage changes in calcium, phosphorus, and calcium-phosphorus product were not reported in detail, a significant decrease in albumincorrected calcium of $\sim 0.6 \mathrm{mg} / \mathrm{dL}$ occurred in both trials. Reported separately, percentage changes in corrected calcium levels in etelcalcetide-treated participants were $-6.7 \%$ and $-7.2 \%$ in trials $\mathrm{A}$ and $\mathrm{B}$, respectively, compared with $1.0 \%$ and $1.2 \%$ in placebo-treated participants. ${ }^{43}$ Serum phosphate was generally reduced in etelcalcetide-treated participants during the course of the trials, although by the trial end, 
only modest differences in phosphate levels between the arms remained. Percentage changes from baseline of serum phosphate levels in etelcalcetide-treated participants were $-9.6 \%$ and $-7.7 \%$ in trials $\mathrm{A}$ and $\mathrm{B}$, respectively, compared with $-1.6 \%$ and $-1.3 \%$ in placebo-treated participants. ${ }^{43}$

Considering results of Phase I and II trials, hypocalcemia was predicted, with implications for how the trials were conducted. While local PTH measurements were suspended during the study period, sites were not blinded to serum calcium values to ensure the safety of study participants. Hypocalcemia below $8.3 \mathrm{mg} / \mathrm{dL}$ occurred in $74.1 \%$ of etelcalcetide-treated participants in trial A and in $82.0 \%$ of etelcalcetide-treated participants in trial B compared with $17.4 \%$ and $21.3 \%$ of respective placebo-treated participants. Corrected calcium below $7.0 \mathrm{mg} / \mathrm{dL}$ occurred in $7.6 \%$ of etelcalcetide-treated participants in the combined trials compared with $3.1 \%$ of placebo-treated participants. Overt symptomatic hypocalcemia was reported in $7.0 \%$ of etelcalcetide-treated participants; the calcium-reducing effect occurred early after treatment initiation and reached a nadir at 10-12 weeks. Hypocalcemia treatment was permitted at the discretion of the treating physician. While use of calcium supplements or calcium-based oral phosphate binders did not appear to differ between arms at baseline, by the end of the trial, the proportion of either or both of these interventions was $\sim 60 \%$ in etelcalcetide-treated participants in trial A but only $\sim 40 \%$ in placebo-treated participants; corresponding values for trial B were $\sim 70 \%$ and $\sim 50 \%$. While the percentages of etelcalcetide-treated participants using activated vitamin $\mathrm{D}$ therapies at baseline were slightly higher in both trials, differences in percentage use tended to widen as the trials progressed; percentages of etelcalcetide-treated participants using vitamin D analogs tended to increase in both trials, and percentages of placebo-treated participants tended to decrease (trial A) or remain stable (trial B).

A general shift toward higher dialysate calcium concentrations occurred over the course of the trials in etelcalcetidetreated participants but not in placebo-treated participants. At the end of trials A and B, respectively, $37.0 \%$ and $51.2 \%$ of etelcalcetide-treated participants were dialyzing on calcium baths $>2.5 \mathrm{mEq} / \mathrm{L}$ compared with only $22.8 \%$ and $29.0 \%$ of placebo-treated participants.

FGF23 levels decreased $\sim 60 \%$ at weeks 12 and 27 in etelcalcetide-treated participants in both trials (with no major change in placebo-treated participants), but final levels were not statistically different between arms in either trial, possibly due to skew in the values. Additionally, markers of bone resorption decreased; serum alkaline phosphate levels in etelcalcetide-treated participants decreased $43.2 \%$ and $47.3 \%$ in trials $\mathrm{A}$ and $\mathrm{B}$, respectively, compared with $12.1 \%$ and $9.7 \%$ in placebo-treated participants, while collagen type I cross-linked C-telopeptide levels in etelcalcetide-treated participants decreased $58.4 \%$ and $63.3 \%$, versus $20 \%$ and $21.5 \%$, in trials A and B, respectively.

Nonclinical safety data indicated no significant concerns for human risk beyond hypocalcemia and the associated prolonged QT interval. ${ }^{44}$ However, the effect of etelcalcetide on the QT interval deserves particular scrutiny. The trials provided a rare and perhaps unprecedented opportunity to examine abnormalities of the QT interval in maintenance hemodialysis patients. Preintervention assessments provided information on the prevalence of QT abnormalities at baseline. Additionally, the phenomena of within- and betweenpatient variation in QT interval could be studied over time as placebo-treated participants progressed through the study, and the effect on QT intervals of a hypocalcemia-inducing drug could be studied by comparing participants randomized to etelcalcetide with those who were not. Electrocardiograms (ECGs) were rigorously performed, in triplicate, at four time points: baseline, twice during the dose-adjustment phase, and once during the efficacy assessment phase. The corrected QT (QTc) interval was assessed in two major ways.

At baseline, substantial QTc prolongation was present. Using the Bazett method (by far the most commonly used method in clinical practice), QTc was $>450-480 \mathrm{~ms}$ for $\sim 25.0 \%$ of all participants, $>480-500 \mathrm{~ms}$ for $5.6 \%$ of participants, and $>500 \mathrm{~ms}$ for $2.7 \%$ of participants before randomization. Corresponding values using an alternative Fridericia method were $13.6 \%, 3.2 \%$, and $0.8 \%$, respectively. A large number of placebo-treated participants had at least one episode of prolonged QTc interval. Using the Bazett method, the maximum absolute post-baseline QTc interval of $>450-480 \mathrm{~ms}$ was observed in $33.1 \%$ of participants, $>480-500 \mathrm{~ms}$ was observed in $8.2 \%$ of participants, and $>500 \mathrm{~ms}$ was observed in $5.5 \%$ of participants. Corresponding values with the Fridericia method were lower, at $19.9 \%$, $5.5 \%$, and $2.1 \%$, respectively, but still relatively high. Fully $8.0 \%$ of participants had a maximum increase (relative to baseline) of $>30 \mathrm{~ms}$ using the Bazett method.

These findings reflect how common prolonged QTc intervals are in maintenance dialysis patients and the apparent high degree of QTc variability in this population, raising doubts about the ability of a single ECG to provide clinically useful information about QT interval abnormalities over time in dialysis patients. Moreover, the effects of drug-induced hypocalcemia resulted in an increased degree and frequency 
of QT-interval abnormalities. In etelcalcetide-treated participants, an increase in the QTc using both methods was observed. A maximum post-baseline QTc interval of $>450$ $480 \mathrm{~ms}$ was observed in $36.0 \%$ of participants, a maximum of $>480-500 \mathrm{~ms}$ in $14.7 \%$ of participants, and a maximum of $>500 \mathrm{~ms}$ in $9.3 \%$ of participants using the Bazett method. A higher percentage of etelcalcetide-treated participants experienced a maximum increase in QTc from baseline; 19.5\% of etelcalcetide-treated participants experienced an increase of $>30-60 \mathrm{~ms}$ compared with $8.0 \%$ of placebo-treated participants. Furthermore, $2.4 \%$ of participants experienced a $>60 \mathrm{~ms}$ increase compared with none of the placebo-treated participants. Overall, using the Bazett method, $60.0 \%$ of etelcalcetide-treated participants experienced a maximum post-baseline QTc $>450 \mathrm{~ms}$ (QTc $>450-480 \mathrm{~ms}$ in $36.0 \%$ of participants, $>480-500 \mathrm{~ms}$ in $14.7 \%$ of participants, and $>500 \mathrm{~ms}$ in $9.3 \%$ of participants).

Death rates were no different between arms in either trial. Death occurred in $2.8 \%$ of etelcalcetide-treated participants in trial $\mathrm{A}$ and $1.6 \%$ of etelcalcetide-treated participants in trial B and in $2.8 \%$ and $3.1 \%$, respectively, of placebo-treated participants.

Given the well-established gastrointestinal side effects of cinacalcet, nausea, vomiting, and diarrhea as potential effects of etelcalcetide treatment were afforded particular scrutiny. The mechanism of gastrointestinal side effects (local in the gastrointestinal tract versus central in the nervous system) of cinacalcet is not fully understood, and it was hypothesized that etelcalcetide may be more readily tolerated, at least with regard to gastrointestinal side effects. Rates of these effects were consistently higher in etelcalcetide-treated participants; $10.7 \%$ of pooled participants reported nausea, $10.7 \%$ reported diarrhea, and $8.9 \%$ reported vomiting compared with $6.2 \%, 8.6 \%$, and $5.1 \%$ of respective placebo-treated participants.

Another Phase III trial was conducted in a Japanese hemodialysis population. ${ }^{45}$ Compared with the study by Block et al, ${ }^{41}$ the Japanese study was smaller (155 participants) and shorter in duration (12 weeks). The primary end point was the proportion of patients who achieved a serum PTH of 60-240 pg/mL (the target range suggested by the Japanese Society for Dialysis Therapy guidelines), ${ }^{46}$ and the proportion of patients with a $>30 \%$ reduction in PTH was the secondary end point. Etelcalcetide was initiated at $5 \mathrm{mg}$ and adjusted in 4-week intervals to doses ranging from 2.5 to $15 \mathrm{mg}$. A baseline PTH of $\geq 300 \mathrm{pg} / \mathrm{mL}$, an albumin-corrected calcium of $\geq 8.4 \mathrm{mg} / \mathrm{dL}$, and a dialysate calcium of $\geq 2.25 \mathrm{mEq} / \mathrm{L}$ were required. As is characteristic of the prevalent Japanese hemodialysis population, participants had a relatively long dialysis duration (11.9 years). Mean PTH at baseline was $536 \mathrm{pg} / \mathrm{mL}$.

The trial demonstrated clear superiority for the intervention; $59.0 \%$ of etelcalcetide-treated participants achieved the goal PTH compared with only $1.3 \%$ of placebo-treated participants. Regarding the secondary end point, $79.6 \%$ of etelcalcetide-treated participants experienced a $>30 \%$ reduction in PTH compared with $5.2 \%$ of placebo-treated participants. Sustained decreases of $\sim 1.0 \mathrm{mg} / \mathrm{dL}$ in serum calcium and phosphate were observed during the study period. Drug-related adverse events occurred in $19.2 \%$ of the etelcalcetide-treated participants and in $3.9 \%$ of placebo-treated participants, with none reported as serious; asymptomatic hypocalcemia (6.4\%), vomiting $(3.8 \%)$, nausea $(1.3 \%)$, and symptomatic hypocalcemia ( $1.3 \%)$ were reported only in etelcalcetide-treated participants. As in the prior Phase III trial, hypocalcemia-related increases in QTc intervals occurred but no clinically relevant cardiovascular adverse events.

Long-term effects of etelcalcetide in the Japanese dialysis population have been reported. In a multicenter, open-label study of 191 participants with baseline PTH of $>240 \mathrm{pg} / \mathrm{mL}$, participants were administered thrice-weekly etelcalcetide with initial $5 \mathrm{mg}$ doses titrated to between 2.5 and $15 \mathrm{mg}$ to achieve PTH levels of $60-240 \mathrm{pg} / \mathrm{mL}$. Mean administered etelcalcetide dose slowly declined during the 52-week trial, with a final mean dose of only $\sim 5.5 \mathrm{mg}$. Starting from a mean baseline PTH of $473 \mathrm{pg} / \mathrm{mL}$, fully $87.5 \%$ of participants achieved the goal after 52 weeks; mean PTH decreased to $157 \mathrm{pg} / \mathrm{mL}$. Nausea was reported in $4.7 \%$ of participants, vomiting in $9.5 \%$ of participants, and symptomatic hypocalcemia in only $1.1 \%$ of participants. Overall, only $7.4 \%$ of participants discontinued the study due to adverse events. ${ }^{47}$

\section{Clinical comparisons to cinacalcet}

As noted, etelcalcetide cannot be accurately described as an IV form of cinacalcet, and a comparison between the agents is informative. In animal experiments using the 5/6th nephrectomy rat model of chronic uremia, ${ }^{48}$ greater and more sustained reduction in PTH occurred among etelcalcetidetreated than among cinacalcet-treated animals over a 4-week period.

As might be predicted, this issue was tested in human beings, with a Phase III head-to-head trial undertaken to demonstrate non-inferiority of etelcalcetide. ${ }^{41}$ Consistent with other trial designs, the primary end point was the proportion 
of participants with $>30 \%$ reduction from baseline in PTH during the efficacy assessment phase. The trial studied 683 participants with baseline predialysis PTH $>500 \mathrm{pg} / \mathrm{mL}$ and albumin-corrected serum calcium $\geq 8.3 \mathrm{mg} / \mathrm{dL}$ on stable doses of CKD-MBD-related agents (calcium supplements, phosphate binders, and calcitriol or active vitamin D analogs). Participants were randomized to etelcalcetide or cinacalcet and were stratified by region and by screening PTH level ( $<900$ versus $\geq 900 \mathrm{pg} / \mathrm{mL}$ ). Participants randomized to etelcalcetide received a starting dose of $5 \mathrm{mg}$ administered thrice weekly after hemodialysis and were titrated in increments of 2.5 or $5 \mathrm{mg}$ up to $15 \mathrm{mg}$. Participants randomized to cinacalcet received an initial dose of $30 \mathrm{mg}$, with the dose titrated up to $180 \mathrm{mg}$. Doses in both arms were adjusted at 4-week intervals during the 20 -week dose-titration phase; doses were withheld for consecutive levels of PTH $<100 \mathrm{pg} /$ $\mathrm{mL}$ or calcium $<7.5 \mathrm{mg} / \mathrm{dL}$, symptomatic hypocalcemia, or drug-related adverse events. Secondary end points included the proportion of participants with $>50 \%$ and $>30 \%$ reductions in PTH, mean weekly days of self-reported nausea and vomiting over the first 8 weeks, and effects on FGF23 and markers of bone turnover.

In this trial, mean age was $\sim 55$ years, and slightly over half of participants were men. Four in five participants were white and approximately one in six black. Approximately $44 \%$ were on dialysate calcium of $\geq 3.0 \mathrm{mg} / \mathrm{dL}$. Overall, characteristics were generally well randomized; mean baseline PTH levels were $1092 \mathrm{pg} / \mathrm{mL}$ in etelcalcetide-treated participants and $1139 \mathrm{pg} / \mathrm{mL}$ in cinacalcet-treated participants. The median pretreatment doses were $5 \mathrm{mg}$ for etelcalcetide and $51.4 \mathrm{mg}$ daily for cinacalcet.

The primary end point occurred in $68.2 \%$ and $57.7 \%$ of etelcalcetide- and cinacalcet-treated participants, respectively, demonstrating non-inferiority for the novel agent. The proportion of participants with $>50 \%$ reduction in PTH from baseline was $12.2 \%$ higher in absolute terms in etelcalcetide-treated participants (52.2\% versus $40.2 \%)$; similarly, the proportion of participants with $>30 \%$ reduction was $10.5 \%$ greater in absolute terms. Participants in both arms experienced co-interventions during the study period, including increased dialysate calcium and greater use of calcium supplements or calcium-containing phosphate binders and calcitriol or active vitamin D analogs; the proportion of etelcalcetide-treated participants who were prescribed these co-interventions was slightly higher. Of note, levels of FGF23 and markers of bone turnover were reduced more in etelcalcetide-treated participants than in cinacalcet-treated participants. At the end of the efficacy assessment phase, $74.4 \%$ of etelcalcetide-treated and $57.5 \%$ of cinacalcet-treated participants achieved $>30 \%$ reduction in FGF23 relative to baseline.

Death occurred in $2.7 \%(n=9)$ of etelcalcetide-treated and in $1.8 \%(n=6)$ of cinacalcet-treated participants; statistically not a significant difference. Perhaps unexpectedly, rates of self-reported nausea and vomiting did not differ significantly, with 0.4 mean adjusted weekly days among etelcalcetide-treated participants and 0.3 mean adjusted weekly days among cinacalcet-treated participants; however, assessment of these side effects occurred only during the first 8 weeks of the study. Overall, nausea and vomiting were common, with comparable percentages in the study arms; nausea occurred in $18.3 \%$ and $22.6 \%$ and vomiting in $13.3 \%$ and $13.8 \%$ of etelcalcetide-treated participants and cinacalcet-treated participants, respectively.

Clinical use of cinacalcet is associated with multiple potential side effects and complications. Tolerance is often limited by adverse effects such as nausea and vomiting, which may well have contributed to the high dropout rate of cinacalcet-treated patients in the EVOLVE trial. Some of these challenges contributing to suboptimal use of cinacalcet can be circumvented with adjustments in medication administration timing during the day and with meals. ${ }^{49}$ Etelcalcetide may allow greater compliance, compared with cinacalcet, with acknowledgement of the high pill burden and potentially complex administration instructions. Widespread use and studies of tolerability in the real-world setting will help inform of changes in tolerability.

\section{Implications of etelcalcetide use}

As with any novel agent, many questions remain about the potential risks and benefits of etelcalcetide. Given the relatively frequent occurrence of hypocalcemia in its Phase III trials, close monitoring of calcium and ready availability of a hypocalcemia mitigation strategy are essential. During the randomized trials, treating physicians often prescribed higher calcium dialysate baths, increased doses of vitamin D analogs, and increased calcium supplementation, such as with calciumbased oral phosphate binder therapy. In addition, prudence is required for patients with known long QT intervals or those at risk of acquired QT prolongation from hypokalemia, hypomagnesemia, hypocalcemia, structural heart disease, or bradyarrhythmias. In addition, individuals taking QT-prolonging drugs, such as antiarrhythmics, antipsychotics, antimicrobials, antiemetics, or antimotility agents, are likely to be at particular risk, meaning that a careful review of a patient's medication list should be undertaken prior to etelcalcetide initiation. 
Although the Phase III trials of etelcalcetide demonstrated large degrees of QT variability in dialysis patients (with the implication that a single ECG reading is likely insufficient to determine risk of QT-interval-related arrhythmia), assessment of QT intervals before initiating etelcalcetide, and review of medications that may affect QT intervals may be appropriate strategies. This could impose a barrier on etelcalcetide prescription, as ECG machines are not readily available in many dialysis units, at least in the US. Whether this degree of caution is warranted, especially if it could deprive a patient of access to a potentially effective PTH-lowering drug, is unknown. To minimize risks in changing from oral to IV calcimimetic, cinacalcet should be discontinued for at least 7 days prior to initiation of etelcalcetide. Serum calcium should be sufficiently high to withstand a hypocalcemic challenge; in our view, a level of at least $8.3 \mathrm{mg} / \mathrm{dL}$, as was used in the clinical trials, seems an appropriate starting point. As per the 2017 KDIGO CKD-MBD guidelines, mild and asymptomatic hypocalcemia can likely be tolerated in the setting of administration of calcimimetics, ${ }^{11}$ provided the benefits of therapy are judged to outweigh the risks.

Regarding potential benefit, etelcalcetide may reduce PTH without the calcium and phosphate loading associated with use of activated vitamin D compounds. Given concerns about calcium loading in CKD and end-stage renal disease, whether etelcalcetide can modify the risk of vascular and soft tissue calcification remains to be seen. There is reason for optimism on this front; cinacalcet has been shown to attenuate vascular calcification, ${ }^{50}$ and etelcalcetide, in a CKD rat model, prevented vascular calcification compared with placebo and paricalcitol..$^{51}$ Additional benefits of calcimimetics include an association with reduced PTX, such as in the EVOLVE trial. ${ }^{10}$ With the potential for a greater and more sustained reduction in PTH than cinacalcet provides, etelcalcetide, if used widely, might result in a reduction in the rate of PTX, although it seems unlikely that etelcalcetide will ever be the subject of a clinical trial akin to EVOLVE. At the same time, one potential risk with etelcalcetide use is a dramatic and sustained PTH lowering, which could lead to the induction of a dynamic bone disease. While this could, in theory, occur with use of any PTH-lowering therapy, these risks may possibly be greater with a potent PTH-lowering agent such as etelcalcetide.

One underappreciated area of investigation is quality of life (QoL), an important aspect to study whenever novel therapeutic interventions are undertaken. This is particularly important in the case of patients receiving maintenance hemodialysis, for whom the symptoms of both the disease and its treatment (hemodialysis) typically prove very burdensome. SHPT itself is associated with multiple systemic symptoms, notably musculoskeletal in nature, which can adversely affect QoL. As a clinical trial of PTX versus calcimimetics has never been performed, the opportunity to rigorously compare these therapeutic approaches for their effects on QoL has never been afforded. However, in uncontrolled studies, PTX has been associated with improved QoL relative to cinacalcet. ${ }^{52}$ It will therefore be important to evaluate the role that etelcalcetide might play in patients' self-assessed QoL as the drug becomes more widely used.

\section{Proposed clinical scenarios for etelcalcetide initiation}

A proposed conceptual approach for initiation of etelcalcetide is given in Tables 1 and 2. Two parent scenarios are given, one in which the PTH level is frankly elevated (e.g., $>600 \mathrm{pg} / \mathrm{mL}$ ) and the other in which the PTH level is within a commonly accepted goal range (e.g., 300-600 pg/mL).

\section{Elevated PTH level}

In all cases, the rationale for etelcalcetide initiation in the setting of hypocalcemia is poor. In the setting of PTH level $>600 \mathrm{pg} / \mathrm{mL}$, normal phosphorus level, and hypercalcemia, etelcalcetide is an attractive option given the possibility that its use will reduce circulating calcium levels while assisting with PTH control. The setting in which all three circulating analytes are increased presents the strongest rationale for etelcalcetide use. When calcium is in the normal range but PTH and phosphorous are elevated, initiation of etelcalcetide might reduce circulating phosphorus levels, but special care must be taken given the possibility of hypocalcemia; if this occurs, co-interventions, such as increasing the dialysate bath calcium concentration, substituting calcium-containing oral phosphate binders for calcium-free ones, increasing doses of activated vitamin D compounds, or otherwise supplying exogenous calcium, are potential approaches. The setting of elevated PTH levels but normocalcemia and normophosphatemia presents at least two options; one involves increasing the dose of an activated vitamin D compound and initiating etelcalcetide if and when phosphorus levels rise, and the other involves initiating etelcalcetide in the absence of hyperphosphatemia (decreasing activated vitamin D dose if PTH decreases and normocalcemia is maintained or increasing activated vitamin $\mathrm{D}$ dose if calcium decreases into a potentially dangerous range). 
Table I Proposed conceptual approach for initiation of etelcalcetide, PTH $>600 \mathrm{pg} / \mathrm{mL}$

\begin{tabular}{|c|c|c|c|}
\hline \multirow{3}{*}{$\frac{\xi}{\frac{1}{u}} \frac{\tilde{J}}{0}$} & High & $\begin{array}{l}\text { Initiate etelcalcetide while adjusting doses of I,25D and other sources } \\
\text { of calcium }\end{array}$ & $\begin{array}{l}\text { Initiate etelcalcetide and adjust I,25D dose } \\
\text { as needed }\end{array}$ \\
\hline & Normal & $\begin{array}{l}\text { Options: } \\
\text { I. Increase } 1,25 \mathrm{D} \text { dose; if hyperphosphatemia results, reduce dose and } \\
\text { initiate etelcalcetide } \\
\text { 2. Initiate etelcalcetide if on high-dose } 1,25 \mathrm{D}^{\mathrm{a}}\end{array}$ & $\begin{array}{l}\text { Initiate etelcalcetide }{ }^{\mathrm{a}} \text { and adjust I,25D dose } \\
\text { as needed }\end{array}$ \\
\hline & Low & \multicolumn{2}{|l|}{ Avoid initiation of etelcalcetide } \\
\hline & & Normal & High \\
\hline & & \multicolumn{2}{|l|}{ Phosphorus } \\
\hline
\end{tabular}

Notes: aMonitor very closely for occurrence of hypocalcemia; if it develops, consider I) increasing dialysate bath calcium concentration; 2) changing from calcium-containing to non-calcium-containing oral phosphorus binders; and 3) prescribing other calcium supplementation. I,25D is calcitriol.

Abbreviation: PTH, parathyroid hormone.

Table 2 Proposed conceptual approach for initiation of etelcalcetide, PTH $\approx 300-600 \mathrm{pg} / \mathrm{mL}$

\begin{tabular}{|c|c|c|c|}
\hline \multirow{3}{*}{$\frac{\xi}{\frac{\delta}{J}}$} & High & $\begin{array}{l}\text { Initiate etelcalcetide while adjusting doses of I,25D and other } \\
\text { sources of calcium }\end{array}$ & Initiate etelcalcetide and adjust I,25D dose as needed \\
\hline & Normal & Initiate etelcalcetide only if on high-dose $I, 25 D^{a}$ & $\begin{array}{l}\text { Reduce I,25D dose if possible; initiate etelcalcetide if } \\
\text { PTH rises }\end{array}$ \\
\hline & Low & \multicolumn{2}{|l|}{ Avoid initiation of etelcalcetide } \\
\hline & & Normal & High \\
\hline & & \multicolumn{2}{|c|}{ Phosphorus } \\
\hline
\end{tabular}

Notes: aMonitor very closely for occurrence of hypocalcemia; if it develops, consider I) increasing dialysate bath calcium concentration; 2) changing from calcium-containing to non-calcium-containing oral phosphorus binders; and 3) prescribing other calcium supplementation. I,25D is calcitriol.

Abbreviation: PTH, parathyroid hormone.

\section{“Controlled” PTH level}

As mentioned earlier, the rationale for etelcalcetide initiation in the setting of hypocalcemia is poor and likely ill-advised. Even in the setting of controlled PTH levels (e.g., 300$600 \mathrm{pg} / \mathrm{mL}$ ), etelcalcetide initiation is a promising option when calcium levels are high, especially when coupled with hyperphosphatemia. In the setting of hyperphosphatemia and normocalcemia, reduction of activated vitamin D dose, coupled with etelcalcetide initiation if required, represents a strategy in which phosphorus levels may be reduced while PTH control is maintained. In normocalcemic patients, the possibility of etelcalcetide-induced hypocalcemia must be carefully monitored. In the setting of normocalcemia and normophosphatemia, etelcalcetide likely has a very limited role, perhaps confined to patients on extremely high doses of activated vitamin $\mathrm{D}$ (an uncommon setting).

\section{Conclusion}

The availability of a second-generation calcimimetic, etelcalcetide, provides treating physicians with a novel agent that can potently reduce PTH levels. That it will be directly administered by hemodialysis personnel should address adherence concerns that characterize use of oral agents. Other potential, but as yet unproven, benefits of etelcalcetide, relative to activated vitamin $\mathrm{D}$ compounds, include reduction in vascular calcification and calcific uremic arteriolopathy (calciphylaxis), decreased risk of need for PTX, reduction in circulating levels of phosphorus and FGF23, and improvement in circulating markers of bone turnover relative to activated vitamin D-based approaches. However, given the partial disappointment associated with the EVOLVE trial, ${ }^{18}$ the nephrology community may have to find solace in these improved surrogate end points, because a trial akin to EVOLVE is unlikely to be repeated with etelcalcetide.

Despite promising signals from the clinical trials, however, whether and how etelcalcetide will be used in real-world clinical scenarios is uncertain. Results to date suggest that etelcalcetide may be associated with levels of gastrointestinal adverse events similar to cinacalcet, which is likely to disappoint many practitioners. However, some initial anecdotal evidence appears to suggest that, in "real-world" clinical scenarios, etelcalcetide may have improved tolerability compared with clinical trial environments; more formal study of this issue is required. Perhaps the problem likely to require the most scrutiny concerns the role of etelcalcetide-induced hypocalcemia and its effect on QTc prolongation. Given the frequency of cardiac arrhythmias and persistently high rates of sudden cardiac death in dialysis patients, ${ }^{53}$ etelcalcetide use in real-world clinical scenarios will require scrutiny by prescribers and, perhaps, more formally in postmarketing studies.

The availability of etelcalcetide expands the armamentarium through which nephrologists can treat SHPT and 
provides an exciting new option to treat PTH elevations. However, additional evidence concerning etelcalcetide's benefits and harms will doubtless appear as it becomes more widely used in clinical practice.

\section{Key points}

1. As demonstrated in large, rigorously conducted randomized clinical trials, etelcalcetide is an effective agent for reducing PTH, providing nephrologists with an alternative to vitamin $\mathrm{D}$ analogs and cinacalcet in maintenance hemodialysis patients with SHPT.

2. Use of etelcalcetide is associated with decreased levels of phosphorous and FGF23 and with an apparently favorable profile regarding markers of bone metabolism.

3. As an IV formulation administered directly into the hemodialysis circuit, etelcalcetide may overcome challenges to adherence that many practitioners observe with cinacalcet.

4. Unfortunately, despite being an IV-administered agent with a long half-life, etelcalcetide was associated with substantial rates of adverse gastrointestinal events in clinical trials, which may be a threat to medication use and adherence.

5. Hypocalcemia with etelcalcetide administration was relatively common, often leading to need for co-interventions such as increased calcium dialysate concentration, calcium-based oral phosphate binders, oral calcium supplementation, and dosing of vitamin D analogs.

6. Prolonged QT intervals may occur with etelcalcetide administration. The clinical significance of this is unknown, but it must be carefully monitored by the treating physician. Serum calcium should be measured before and during treatment, and practitioners should be aware of potential effects of QT interval prolongation.

7. The overall effect of etelcalcetide on calcium balance remains unknown, and co-interventions designed to counteract etelcalcetide-induced hypocalcemia may be required in many patients.

8. The potential effects of etelcalcetide on intermediate (surrogate) end points, such as vascular calcification burden, bone phenotype, and fracture rates, are unknown, as are potential associations of etelcalcetide with improvements in all-cause or cardiovascular mortality or QoL.

\section{Acknowledgment}

The authors thank Chronic Disease Research Group's colleagues Anne Shaw for manuscript preparation and Nan Booth, MSW, MPH, ELS, for manuscript editing.

\section{Disclosure}

KEE has received honoraria from Amgen. JBW reports no conflicts of interest. The authors report no other conflicts of interest in this work.

\section{References}

1. Kidney Disease: Improving Global Outcomes (KDIGO) CKD-MBD Work Group. KDIGO clinical practice guideline for the diagnosis, evaluation, prevention, and treatment of chronic kidney disease-mineral and bone disorder (CKD-MBD). Kidney Int Suppl. 2009;76(Suppl 113):S1-S130.

2. Block GA, Hulbert-Shearon TE, Levin NW, Port FK. Association of serum phosphorus and calcium $\mathrm{x}$ phosphate product with mortality risk in chronic hemodialysis patients: a national study. Am J Kidney Dis. 1998;31(4):607-617.

3. Block GA, Klassen PS, Lazarus JM, Ofsthun N, Lowrie EG, Chertow GM. Mineral metabolism, mortality, and morbidity in maintenance hemodialysis. J Am Soc Nephrol. 2004;15(8):2208-2218.

4. Tentori F, Wang M, Bieber BA, et al. Recent changes in therapeutic approaches and association with outcomes among patients with secondary hyperparathyroidism on chronic hemodialysis: the DOPPS study. Clin J Am Soc Nephrol. 2015;10(1):98-109.

5. Cunningham J, Locatelli F, Rodriguez M. Secondary hyperparathyroidism: pathogenesis, disease progression, and therapeutic options. Clin J Am Soc Nephrol. 2011;6(4):913-921.

6. Nickolas TL, Leonard MB, Shane E. Chronic kidney disease and bone fracture: a growing concern. Kidney Int. 2008;74(6):721-731.

7. Hruska KA, Choi ET, Memon I, Davis TK, Mathew S. Cardiovascular risk in chronic kidney disease (CKD): the CKD-mineral bone disorder (CKD-MBD). Pediatr Nephrol. 2010;25(4):769-778.

8. Faul C, Amaral AP, Oskouei B, et al. FGF23 induces left ventricular hypertrophy. J Clin Invest. 2011;121(11):4393-4408.

9. Moe SM, Chertow GM, Coburn JW, et al. Achieving NKF-K/DOQI bone metabolism and disease treatment goals with cinacalcet $\mathrm{HCl}$. Kidney Int. 2005;67(2):760-771.

10. Chertow GM, Block GA, Correa-Rotter R, et al. Effect of cinacalcet on cardiovascular disease in patients undergoing dialysis. $N$ Engl J Med. 2012;367(26):2482-2494

11. Kidney Disease Improving Global Outcomes (KDIGO). KDIGO 2017 Clinical practice guideline update for the diagnosis, evaluation, prevention and treatment of chronic kidney disease-mineral and bone disorder CKD-MBD. Kidney Int Suppl. 2017;7(1):1-59.

12. Palmer SC, McGregor DO, Macaskill P, Craig JC, Elder GJ, Strippoli GF. Meta-analysis: vitamin D compounds in chronic kidney disease. Ann Intern Med. 2007;147(12):840-853.

13. Wetmore JB, Liu S, Krebill R, Menard R, Quarles LD. Effects of cinacalcet and concurrent low-dose vitamin D on FGF23 levels in ESRD. Clin J Am Soc Nephrol. 2010;5(1):110-116.

14. Gutierrez OM, Mannstadt M, Isakova T, et al. Fibroblast growth factor 23 and mortality among patients undergoing hemodialysis. $N$ Engl $J$ Med. 2008;359(6):584-592.

15. St Peter WL, Li Q, Liu J, et al. Cinacalcet use patterns and effect on laboratory values and other medications in a large dialysis organization, 2004 through 2006. Clin J Am Soc Nephrol. 2009;4(2):354-360.

16. Vervloet M, Bencova V, Malberti F, et al. "Real-World" use of cinacalcet for managing SHPT in different European countries: analysis of data from the ECHO observational study. Clin Nephrol. 2010;74(3):198-208.

17. Perkovic V, Neal B. Trials in kidney disease - time to EVOLVE. N Engl J Med. 2012;367(26):2541-2542.

18. Parfrey PS, Block GA, Correa-Rotter R, et al. Lessons learned from EVOLVE for planning of future randomized trials in patients on dialysis. Clin J Am Soc Nephrol. 2016;11(3):539-546.

19. Li S, Chen YW, Peng Y, Foley RN, St Peter WL. Trends in parathyroidectomy rates in US hemodialysis patients from 1992 to 2007. Am J Kidney Dis. 2011;57(4):602-611. 
20. Kim SM, Long J, Montez-Rath ME, Leonard MB, Norton JA, Chertow GM. Rates and outcomes of parathyroidectomy for secondary hyperparathyroidism in the United States. Clin J Am Soc Nephrol. 2016;11(7):1260-1267.

21. Tominaga Y, Kakuta T, Yasunaga C, Nakamura M, Kadokura Y, Tahara H. Evaluation of parathyroidectomy for secondary and tertiary hyperparathyroidism by the Parathyroid Surgeons' Society of Japan. Ther Apher Dial. 2016;20(1):6-11.

22. Ishani A, Liu J, Wetmore JB, et al. Clinical outcomes after parathyroidectomy in a nationwide cohort of patients on hemodialysis. Clin J Am Soc Nephrol. 2015;10(1):90-97.

23. Wetmore JB, Liu J, Do TP, et al. Changes in secondary hyperparathyroidism-related biochemical parameters and medication use following parathyroidectomy. Nephrol Dial Transplant. 2016;31(1):103-111.

24. Subramanian R, Zhu X, Kerr SJ, et al. Nonclinical pharmacokinetics, disposition, and drug-drug interaction potential of a novel D-amino acid peptide agonist of the calcium-sensing receptor AMG 416 (etelcalcetide). Drug Metab Dispos. 2016;44(8):1319-1331.

25. Funke SA, Willbold D. Mirror image phage display - a method to generate D-peptide ligands for use in diagnostic or therapeutical applications. Mol Biosyst. 2009;5(8):783-786.

26. Magno AL, Ward BK, Ratajczak T. The calcium-sensing receptor: a molecular perspective. Endocr Rev. 2011;32(1):3-30.

27. Alexander ST, Hunter T, Walter S, et al. Critical cysteine residues in both the calcium-sensing receptor and the allosteric activator AMG 416 underlie the mechanism of action. Mol Pharmacol. 2015;88(5):853-865.

28. Subramanian R, Zhu X, Hock MB, et al. Pharmacokinetics, biotransformation, and excretion of [14C] etelcalcetide (AMG 416) following a single microtracer intravenous dose in patients with chronic kidney disease on hemodialysis. Clin Pharmacokinet. 2017;56(2):179-192.

29. Wu L, Melhem M, Subramanian R, Wu B. Drug disposition model of radiolabeled etelcalcetide in patients with chronic kidney disease and secondary hyperparathyroidism on hemodialysis. J Pharmacokinet Pharmacodyn. 2017;44(1):43-53.

30. Edson KZ, Wu BM, Iyer A, Goodman W, Skiles GL, Subramanian R. Determination of etelcalcetide biotransformation and hemodialysis kinetics to guide the timing of its dosing. Kidney Int. 2016;1(1):24-33.

31. Kroenke MA, Weeraratne DK, Deng H, et al. Clinical immunogenicity of the D-amino acid peptide therapeutic etelcalcetide: method development challenges and anti-drug antibody clinical impact assessments. J Immunol Methods. 2017;445:37-44.

32. Baruch A, Maclean D, Yin K, et al [webpage on the Internet]. KAI-4169, a novel calcimimetic for the treatment of secondary hyperparathyroidism [abstract]. Presented at the Endocrine Society's 93rd Annual Meeting \& Expo; June 5, 2011, Presentation P2-98; Boston. Available from: http:// press.endocrine.org/doi/abs/10.1210/endo-meetings.2011.PART2.P25. P2-98. Accessed January 17, 2018.

33. Walter S, Baruch A, Dong J, et al. Pharmacology of AMG 416 (Velcalcetide), a novel peptide agonist of the calcium-sensing receptor, for the treatment of secondary hyperparathyroidism in hemodialysis patients. J Pharmacol Exp Ther. 2013;346(2):229-240.

34. Shen J, Xiao J, Pickthorn K, et al. A pharmacokinetic/pharmacodynamic model for AMG 416, a novel calcimimetic peptide, following a single intravenous dose in healthy subjects. J Clin Pharmacol. 2014;54(10): 1125-1133.

35. Martin KJ, Bell G, Pickthorn K, et al. Velcalcetide (AMG 416), a novel peptide agonist of the calcium-sensing receptor, reduces serum parathyroid hormone and FGF23 levels in healthy male subjects. Nephrol Dial Transplant. 2014;29(2):385-392.

36. Martin KJ, Pickthorn K, Huang S, et al. AMG 416 (Velcalcetide) is a novel peptide for the treatment of secondary hyperparathyroidism in a single-dose study in hemodialysis patients. Kidney Int. 2014;85(1): 191-197.
37. Yokoyama K, Fukagawa M, Shigematsu T, et al. A single- and multipledose, multicenter study of etelcalcetide in Japanese hemodialysis patients with secondary hyperparathyroidism. Kidney Int. 2017;2(4): 634-644.

38. Bell G, Huang S, Martin KJ, Block GA. A randomized, double-blind, phase 2 study evaluating the safety and efficacy of AMG 416 for the treatment of secondary hyperparathyroidism in hemodialysis patients. Curr Med Res Opin. 2015;31(5):943-952.

39. Bushinsky DA, Block GA, Martin KJ, et al. Treatment of secondary hyperparathyroidism: results of a phase 2 trial evaluating an intravenous peptide agonist of the calcium-sensing receptor. Am J Nephrol. 2015;42(5): 379-388.

40. Yokoyama K, Fukagawa M, Shigematsu T, et al. A 12-week doseescalating study of etelcalcetide (ONO-5163/AMG 416), a novel intravenous calcimimetic, for secondary hyperparathyroidism in Japanese hemodialysis patients. Clin Nephrol. 2017;88(8):68-78.

41. Block GA, Bushinsky DA, Cheng S, et al. Effect of etelcalcetide vs cinacalcet on serum parathyroid hormone in patients receiving hemodialysis with secondary hyperparathyroidism: a randomized clinical trial. JAMA. 2017;317(2):156-164.

42. Fishbane S, Shapiro WB, Corry DB, et al. Cinacalcet $\mathrm{HCl}$ and concurrent low-dose vitamin D improves treatment of secondary hyperparathyroidism in dialysis patients compared with vitamin D alone: the ACHIEVE study results. Clin J Am Soc Nephrol. 2008;3(6):1718-1725.

43. Nemeth EF, Goodman WG. Calcimimetic and calcilytic drugs: feats, flops, and futures. Calcif Tissue Int. 2016;98(4):341-358.

44. Fielden MR, Dean C Jr, Black K, et al. Nonclinical safety profile of etelcalcetide, a novel peptide calcimimetic for the treatment of secondary hyperparathyroidism. Int J Toxicol. 2016;35(3):294-308.

45. Fukagawa M, Yokoyama K, Shigematsu T, et al. A phase 3, multicentre, randomized, double-blind, placebo-controlled, parallel-group study to evaluate the efficacy and safety of etelcalcetide (ONO-5163/AMG 416), a novel intravenous calcimimetic, for secondary hyperparathyroidism in Japanese haemodialysis patients. Nephrol Dial Transplant. 2017;32(10):1723-1730.

46. Fukagawa M, Yokoyama K, Koiwa F, et al. Clinical practice guideline for the management of chronic kidney disease-mineral and bone disorder. Ther Apher Dial. 2013;17(3):247-288.

47. Shigematsu T, Fukagawa M, Yokoyama K, et al. Long-term effects of etelcalcetide as intravenous calcimimetic therapy in hemodialysis patients with secondary hyperparathyroidism. Clin Exp Nephrol. Epub 2017 Aug 23.

48. Walter S, Baruch A, Alexander ST, et al. Comparison of AMG 416 and cinacalcet in rodent models of uremia. BMC Nephrol. 2014;15:81.

49. Bover J, Urena P, Ruiz-Garcia C, et al. Clinical and Practical Use of Calcimimetics in Dialysis Patients With Secondary Hyperparathyroidism. Clin J Am Soc Nephrol. 2016;11(1):161-174.

50. Raggi P, Chertow GM, Torres PU, et al. The ADVANCE study: a randomized study to evaluate the effects of cinacalcet plus low-dose vitamin D on vascular calcification in patients on hemodialysis. Nephrol Dial Transplant. 2011;26(4):1327-1339.

51. Yu L, Tomlinson JE, Alexander ST, et al. Etelcalcetide, a novel calcimimetic, prevents vascular calcification in a rat model of renal insufficiency with secondary hyperparathyroidism. Calcif Tissue Int. 2017;101(6):641-653.

52. van der Plas WY, Dulfer RR, Engelsman AF, et al. Effect of parathyroidectomy and cinacalcet on quality of life in patients with end-stage renal disease-related hyperparathyroidism: a systematic review. Nephrol Dial Transplant. 2017;32(11):1902-1908.

53. Wetmore JB, Li S, Molony JT, et al. Insights from the 2016 peer kidney care initiative report: still a ways to go to improve care for dialysis patients. Am J Kidney Dis. 2018;71(1):123-132. 
The International Journal of Nephrology and Renovascular Disease is an international, peer-reviewed open access journal focusing on the pathophysiology of the kidney and vascular supply. Epidemiology, screening, diagnosis, and treatment interventions are covered as well as basic science, biochemical and immunological studies. The manuscript management system is completely online and includes a very quick and fair peer-review system, which is all easy to use. Visit http://www. dovepress.com/testimonials.php to read real quotes from published authors.

Submit your manuscript here: https://www.dovepress.com/international-journal-of-nephrology-and-renovascular-disease-journal 